\title{
Efficient Denoising of Images with Smooth Geometry
}

\author{
Agnieszka Lisowska \\ University of Silesia, Institute of Informatics, \\ ul. Bedzinska 39, 41-200 Sosnowiec, Poland \\ alisow@ux2.math.us.edu.pl \\ http://www.math.us.edu.pl/al/eng_index.html
}

\begin{abstract}
In the paper the method of smooth geometry image denoising has been presented. It is based on smooth second order wedgelets proposed in this paper. Smooth wedgelets (and second order wedgelets) are defined as wedgelets with smooth edges. Additionally, smooth borders of quadtree partition have been introduced. The first kind of smoothness is defined adaptively whereas the second one is fixed once for the whole estimation process. The proposed kind of wedgelets has been applied to image denoising. As follows from experiments performed on benchmark images this method gives far better results of denoising of images with smooth geometry than the other state-of-the-art methods.
\end{abstract}

Keywords: Image denoising, wedgelets, second order wedgelets, smooth edges, multiresolution.

\section{Introduction}

Image denoising plays very important role in image processing. It follows from the fact that images are obtained mainly from different electronic devices. It causes that many kinds of noise generated by these devices are present on such images. It is well known fact that medical images are characterized by Gaussian noise and astronomical images are corrupted by Poisson noise, to mention a few kinds of noise. Determination of the noise characteristic is not difficult and may be done automatically. The main problem is related to defining of efficient methods of image denoising.

In the case of the most commonly generated Gaussian noise there is a wide spectrum of denoising methods. These methods are based on wavelets due to the fact that noise is characterized by high frequency what can be suppressed just by wavelets. Image denoising by wavelets is very similar to compression in order to perform denoising a forward transform is applied, some coefficients are replaced by zero and then the inverse transform is applied [1. The standard method was improved in many ways, to mention an introduction of different kinds of thresholds or different kinds of thresholding [2, [3].

Recently, also geometrical wavelets have been introduced to image denoising. Since they give better results in image coding than classical wavelets they are also 
applied in image estimation. There is a wide spectrum of geometrical wavelets, for example the ones based on frames like ridgelets [4, curvelets [5], bandelets [6], or the ones based on dictionaries like wedgelets [7, beamlets [8, platelets 9 . As presented in the literature [10, [11, 12] some of them give better results of image denoising than classical wavelets. Especially, in [12] a comparative study is presented from which follows that adaptive methods based on wedgelets are competitive in image denoising to the other wavelets-based methods.

In the paper the improvement of wedgelets-based technique of image estimation has been proposed. It was motivated by the observation that edges present in images are of different smoothness kind. Because second order wedgelets always estimate smooth edges by sharp step functions it may introduce additional errors. In order to avoid it second order wedgelets with smooth edges and smooth borders are used in image denoising. From the experiments performed on the set of benchmark images follows that the proposed method assures better results of image denoising than the leading state-of-the-art methods.

\section{Geometrical Image Denoising}

The problem of image denoising is related to image estimation. Instead of approximating of original image $F$ one needs to estimate it basing only on a version contaminated by noise

$$
I\left(x_{1}, x_{2}\right)=F\left(x_{1}, x_{2}\right)+\sigma Z\left(x_{1}, x_{2}\right), \quad x_{1}, x_{2} \in[0,1],
$$

where $Z$ is an additive zero-mean Gaussian noise. Image $F$ can be quite effectively estimated by multiresolution techniques thanks to the fact that a frequency of added noise is usually higher than that of the original image.

\subsection{Multiresolution Denoising}

The great majority of denoising methods is based on wavelets. It follows from the fact that wavelets are efficient in removing high frequency signal (especially a noise) from an image. However, these methods tend to slightly smoothen edges present on images. So, similarly as in the case of image coding, geometrical wavelets have been introduced to image estimation. Thanks to the possibility of catching changes of signal in different directions they are more efficient in image denoising than classical wavelets. For example, the denoising method based on curvelets [10] is characterized by very efficient estimation nearby edges giving very accurate denoising results. However, as shown in [11], [12, adaptive geometrical wavelets can assure even better estimation results than curvelets. The methods based on wedgelets [11] or second order wedgelets [12] are very efficient in proper reconstruction of image geometry. Below we describe the wedgeletsbased methods in more details.

\subsection{Geometrical Denoising}

Consider an image $F$ defined on dyadic discrete support of size $N \times N$ pixels (dyadic means that $N=2^{n}$ for $n \in \mathbb{N}$ ). To such an image a quadtree partition 
may be assigned. Consider then any square $S$ from that partition and any line segment $b$ (called beamlet [8]) connecting any two points (not lying on the same border side) from the border of the square. The wedgelet is defined as 7 ]

$$
W(x, y)=\mathbf{1}\{y \leq b(x)\}, \quad(x, y) \in S
$$

Similarly, consider any segment of second degree curve (as ellipse, parabola or hyperbola) $\hat{b}$ (called second order beamlet [13, [14]) connecting any two points from the border of the square $S$. The second order wedgelet is defined as [13], 14

$$
\hat{W}(x, y)=\mathbf{1}\{y \leq \hat{b}(x)\}, \quad(x, y) \in S .
$$

Taking into account all possible squares from the quadtree partition (of different locations and scales) and all possible beamlet connections one obtains the wedgelets' dictionary. Taking additionally all possible curvatures of second order beamlets one obtains the second order wedgelets' dictionary. It is assumed that the wedgelets' dictionary is included in the second order wedgelets' dictionary (with the parameter reflecting curvature equals to zero). Because wedgelet is a special case of second order wedgelet in the rest of the paper the dictionary of second order wedgelets is considered. Additionally, second order wedgelets are called for simplicity as s-o wedgelets.

Such defined set of functions can be used adaptively in image approximation or estimation. It is performed in the way that s-o wedgelets are adapted to a geometry from an image. Depending on image content appropriate s-o wedgelets are used in approximation. The process is performed in two steps. In the first step a so-called full quadtree is built. Each node of the quadtree represents the best s-o wedgelet within the appropriate square in the mean of Mean Square Error (MSE) sense. In the second step the tree is pruned in order to solve the following minimization problem

$$
P=\min \left\{\left\|F-F_{\hat{W}}\right\|_{2}^{2}+\lambda^{2} K\right\},
$$

where minimum is taken within all possible quad-splits of an image, $F$ denotes the original image, $F_{\hat{W}}$ its s-o wedgelet approximation, $K$ the number of s-o wedgelets used in approximation and $\lambda$ is the penalization factor. Indeed, we are interested in obtaining the sparsest image approximation assuring the best quality in the mean of MSE metric sense of the approximation. The minimization problem can be solved by the use of the bottom-up tree pruning algorithm 7 .

The algorithm of s-o wedgelets-based image denoising is very similar to image approximation. The only difference is that instead of original image approximation a noised image approximation is performed. However, the additional problem has to be solved. Because the approximation algorithm is dependent on parameter $\lambda$, the optimal value of it should be obtained. It is done in the way that the second step of the approximation is repeated for different values of $\lambda$. As the optimal one is chosen the one for which the dependency between $\lambda$ and number of s-o wedgelets has a saddle point [12]. 


\section{$3 \quad$ Image Denoising with Smooth Wedgelets}

Many images are characterized by presence of edges with different kinds of smoothness. In the case of artificial images very often edges are rather sharp and well defined. However, in the case of still images some edges are sharp and the others are more or less smooth. The approximation of smooth edges by s-o wedgelets causes that MSE increases. It leads to false edges detection what degenerates denoising results. However, the problem may be solved by introducing smooth s-o wedgelets.

\subsection{Smooth Wedgelets Denoising}

Consider any s-o wedgelet like the ones presented in Fig. 1 (a), (c). Smooth s-o wedgelet is defined by introducing smooth connection between two s-o wedgelets defined within the square support (see Fig. 1 (b), (d)). In other words, instead of step discontinuity we introduce linear continuity between two constant areas represented by s-o wedgelets. In this way we introduce additional parameter to the s-o wedgelets' dictionary. The parameter, denoted as $R$, reflects the half of the length of smoothness of the edge. For $R=0$ we obtain just s-o wedgelet, and the larger the value of $R$ the longer the smoothness. This approach is symmetrical. It means that the smoothness is equally elongated on both sides of the original edge (marked in Fig. 1] (b), (d)).

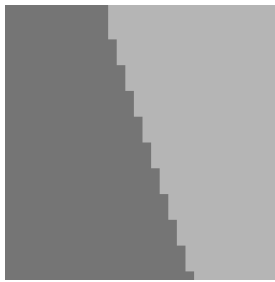

(a)

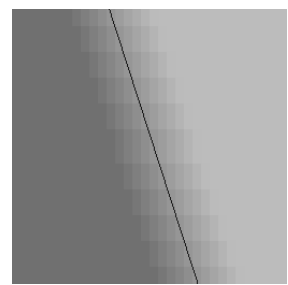

(b)

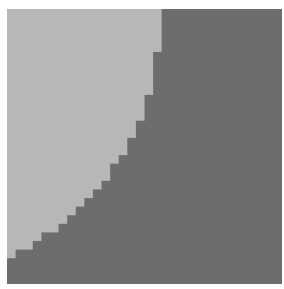

(c)

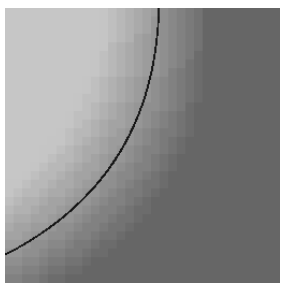

(d)

Fig. 1. (a) Wedgelet, (b) smooth wedgelet, (c) s-o wedgelet, (d) smooth s-o wedgelet

Because wedgelets-based algorithms are known to have large time complexity the additional parameter causes that the computation time is not acceptable. To overcome that problem the following method of finding optimal smooth so wedgelets is proposed. Consider any square $S$ from the quadtree partition. Firstly, find an optimal wedgelet within it. Secondly, basing on it find the best so wedgelet in the neighborhood, like proposed in [14. And finally, basing on the best s-o wedgelet find optimal smooth s-o wedgelet basing on it by incrementing the value of $R$ and computing new values of constant areas. While you find better approximation do incrementation, otherwise stop. This method not necessary assures that the best smooth s-o wedgelet is found but great improvement in the 
approximation is done anyway. After processing of all nodes of the quadtree the bottom-up tree pruning may be applied.

Smooth s-o wedgelets are used in image denoising in the same way as s-o wedgelets are. The algorithm works according to the following steps:

1. Find the best smooth s-o wedgelet matching for every node of the quadtree.

2. Apply the bottom-up tree pruning algorithm to find the optimal approximation.

3. Repeat step 2 for different values of $\lambda$ and choose as the final result the one which gives the best result of denoising.

The most problematic step of the algorithm is to find the optimal value of $\lambda$. In the case of original image approximation the value of $\lambda$ may be set as the one for which RD dependency (in other words the plot of number of wedgelets versus MSE) has the saddle point. Since we do not know the original image we have to use the plot of $\lambda$ versus number of wedgelets and the saddle point of that dependency [1], 12 .

\subsection{Double Smooth Wedgelets}

When we deal with images with smooth geometry we can additionally apply the postprocessing step in order to improve the results of denoising performed by smooth s-o wedgelets. Because all quadtree-based techniques lead to blocking artifacts, especially in smooth images, in the postprocessing step we perform smoothing between neighboring blocks. The length of smoothing is represented by parameter $R_{S}$. It is defined in the same way as parameter $R$. However, the differences between them are meaningful. Indeed, parameter $R$ works in adaptive way, it means that depending on an estimated image its value changes and different values of $R$ lead to different values of wedgelet parameters (represented by constant areas). Typically, different segments of approximated image are characterized by different values of $R$. On the other hand parameter $R_{S}$ is constant and does not depend on the image content. Once fixed for a given image, it never changes.

Taking into account above considerations we can define double smooth s-o wedgelet as a smooth s-o wedgelet with smooth borders. An example of image approximation by such wedgelets is presented in Fig. 2. As one can see the more smoothness is used the better approximation we obtain.

\section{Experimental Results}

The experiments presented in this section were performed on the set of benchmark images presented in Fig. 3. All the described methods were implemented in Borland $\mathrm{C}++$ Builder 6 environment. The images were artificially noised by Gaussian noise with zero mean and eight different values of variances (presented in the paper after normalization). This set of images was submitted to denoising process with the use of three different methods, namely based on wedgelets, s-o 


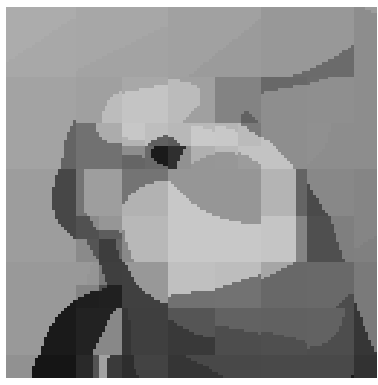

(a)

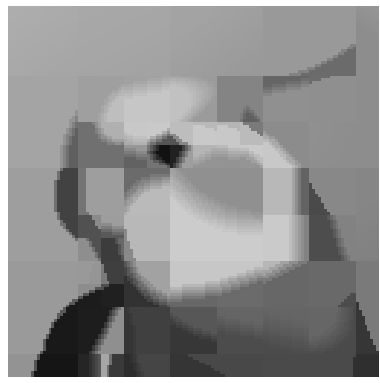

(b)

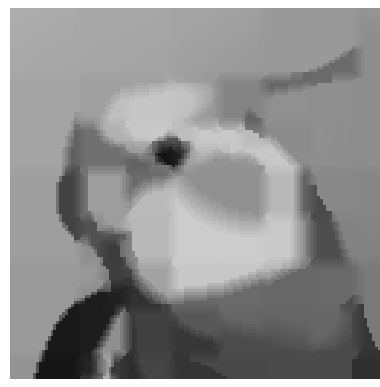

(c)

Fig. 2. The segment of "bird" approximated by (a) s-o wedgelets, (b) smooth s-o wedgelets, (c) double smooth s-o wedgelets

wedgelets and smooth s-o wedgelets (with and without the postprocessing). Additionally, we assumed that $R_{S}=1$. As follows from experiments larger values of $R_{S}$ give better results of denoising only for very smooth images (like "chromosome"). Setting the parameter to one causes that in nearly all tested images an improvement is visible. It should be mentioned also that we applied smooth borders only for square sizes larger than $4 \times 4$ pixels.

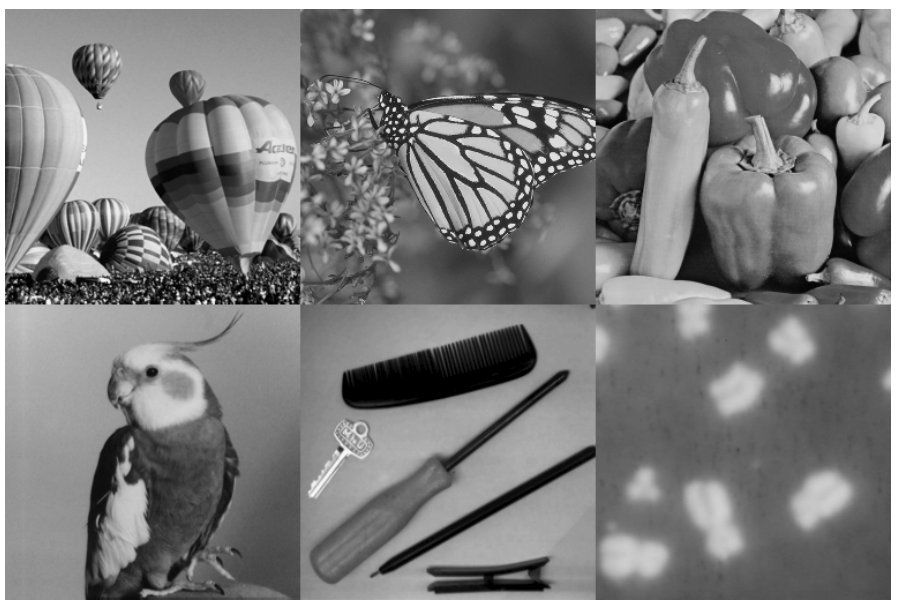

Fig. 3. The benchmark images: "balloons", "monarch", "peppers", "bird", "objects", "chromosome"

In Table 1 the numerical results of image denoising are presented. From that table follows that the proposed method (denoted as wedgelets2S) assures better denoising results than the state-of-the-art reference methods (for further comparisons, like between wavelets and wedgelets see [12]). More precisely, in the case of images without smooth geometry (like "balloons") the improvement of denoising method based on smooth s-o wedgelets is rather small. However, in the 
Table 1. Numerical results of image denoising with the help of the following methods: wedgelets, s-o wedgelets (wedgelets2), smooth s-o wedgelets (wedgelets2S) and double smooth s-o wedgelets (wedgelets2SS)

\begin{tabular}{|c|c|c|c|c|c|c|c|c|}
\hline \multirow[b]{2}{*}{ Image } & \multirow[b]{2}{*}{ Method } & \multicolumn{7}{|c|}{ Noise variance } \\
\hline & & 0.001 & \begin{tabular}{|l|l|}
0.005 & 0.010 \\
\end{tabular} & 0.015 & 0.022 & 0.030 & 0.050 & 0.070 \\
\hline \multirow[t]{4}{*}{ balloons } & wedgelets & 30.50 & \begin{tabular}{|l|l|l}
26.10 & 24.03 \\
\end{tabular} & 23.17 & 22.29 & 21.72 & 20.60 & 19.94 \\
\hline & wedgelets2 & 30.40 & \begin{tabular}{|l|l|}
25.94 .00 & 24.00
\end{tabular} & 23.12 & 22.26 & 21.71 & 20.67 & 19.97 \\
\hline & wedgelets2S & 29.99 & \begin{tabular}{|l|l|}
26.36 & 24.45 \\
\end{tabular} & 23.35 & 22.49 & 21.93 & 20.75 & 20.05 \\
\hline & wedgelets2SS & 29.89 & \begin{tabular}{|l|l|}
26.57 & 24.84 \\
\end{tabular} & 23.80 & 22.98 & 22.44 & 21.24 & 20.42 \\
\hline \multirow[t]{4}{*}{ monarch } & wedgelets & 30.47 & \begin{tabular}{|l|l|}
26.20 & 24.34 \\
\end{tabular} & 23.27 & 22.33 & 21.63 & 20.50 & 19.70 \\
\hline & wedgelets2 & 30.38 & \begin{tabular}{|l|l|}
26.21 & 24.39 \\
\end{tabular} & 23.40 & 22.37 & 21.71 & 20.56 & 19.71 \\
\hline & wedgelets $2 \mathrm{~S}$ & 29.15 & \begin{tabular}{|l|l|}
25.97 & 24.37
\end{tabular} & 23.45 & 22.50 & 21.80 & 20.59 & 19.81 \\
\hline & wedgelets2SS & 28.69 & \begin{tabular}{|l|l|}
25.88 & 24.50 \\
\end{tabular} & 23.71 & 22.91 & 22.23 & 21.02 & 20.29 \\
\hline \multirow[t]{4}{*}{ peppers } & wedgelets & 31.71 & \begin{tabular}{|l|l|}
27.44 & 25.82 \\
\end{tabular} & 24.89 & 24.10 & 23.41 & 22.43 & 21.75 \\
\hline & wedgelets2 & 31.56 & \begin{tabular}{|l|l|}
27.31 & 25.81
\end{tabular} & 24.79 & 24.04 & 23.37 & 22.36 & 21.68 \\
\hline & wedgelets2S & 31.82 & \begin{tabular}{|l|l|}
27.77 & 26.21
\end{tabular} & 25.28 & 24.47 & 23.72 & 22.63 & 21.95 \\
\hline & wedgelets2SS & 31.82 & \begin{tabular}{|l|l|}
28.39 & 26.92 \\
\end{tabular} & 26.03 & 25.11 & 24.36 & 23.11 & 22.35 \\
\hline \multirow[t]{4}{*}{ bird } & wedgelets & 34.24 & \begin{tabular}{|l|l|}
30.24 & 28.76 \\
\end{tabular} & 28.05 & 27.35 & 26.82 & 25.71 & 25.21 \\
\hline & wedgelets2 & 34.07 & \begin{tabular}{|l|l|}
30.24 & 28.76
\end{tabular} & 28.02 & 27.29 & 26.79 & 25.66 & 25.09 \\
\hline & wedgelets $2 \mathrm{~S}$ & 34.61 & \begin{tabular}{|l|l|l|}
30.70 & 29.25
\end{tabular} & 28.54 & 27.74 & 27.24 & 26.01 & 25.38 \\
\hline & wedgelets2SS & 34.90 & \begin{tabular}{|l|l|}
31.41 & 30.00 \\
\end{tabular} & 29.08 & 28.28 & 27.70 & 26.47 & 25.72 \\
\hline \multirow[t]{4}{*}{ objects } & wedgelets & 33.02 & \begin{tabular}{|l|l|}
28.36 & 26.90 \\
\end{tabular} & 25.89 & 25.16 & 24.43 & 23.51 & 22.73 \\
\hline & wedgelets2 & 32.84 & \begin{tabular}{|l|l|}
28.27 & 26.72
\end{tabular} & 25.82 & 25.15 & 24.34 & 23.47 & 22.66 \\
\hline & wedgelets $2 \mathrm{~S}$ & 33.36 & \begin{tabular}{|l|l|}
29.46 & 27.85
\end{tabular} & 26.84 & 25.96 & 25.26 & 24.13 & 23.24 \\
\hline & wedgelets2SS & 33.46 & \begin{tabular}{|l|l|}
29.98 & 28.36 \\
\end{tabular} & 27.41 & 26.43 & 25.69 & 24.51 & 23.51 \\
\hline \multirow[t]{4}{*}{ chromosome } & \begin{tabular}{|l|} 
wedgelets \\
\end{tabular} & 36.45 & \begin{tabular}{|l|l|}
32.78 & 31.48 \\
\end{tabular} & 30.40 & 29.56 & 29.07 & 28.31 & 27.15 \\
\hline & wedgelets2 & 36.29 & \begin{tabular}{|l|l|}
32.69 & 31.31
\end{tabular} & 30.31 & 29.56 & 29.29 & 28.32 & 27.12 \\
\hline & wedgelets $2 \mathrm{~S}$ & 38.00 & \begin{tabular}{|l|l|}
34.67 & 33.24 \\
\end{tabular} & 32.43 & 31.30 & 30.71 & 29.52 & 28.17 \\
\hline & wedgelets2SS & 38.78 & \begin{tabular}{|l|l|}
35.34 & 33.91 \\
\end{tabular} & 33.03 & 31.73 & 31.17 & 29.94 & 28.56 \\
\hline
\end{tabular}

case of images with typical smooth geometry (like "chromosome" and "objects") the improvement is substantial and can oscillate round $1.6 \mathrm{~dB}$. For images with smooth and non-smooth geometry the improvement depends on the amount of smooth geometry within an image.

However, applying the method of denoising based on smooth s-o wedgelets (and wedgelets in general) causes that the so-called blocking artifacts are visible. Even if the denoising results are competitive in the mean of PSNR values the visible false edges lead to uncomfortable perceiving such images by human observer. To overcome that inconvenience also the double smooth s-o wedgelets were applied to image denoising (denoted as wedgelets2SS). As follows from Table 1 that method additionally improves the results of denoising quite substantially.

Additionally, in Fig. 4 the sample result of denoising is presented. As one can see the method based on s-o wedgelets introduces false edges in the very smooth image. Applying smooth s-o wedgelets causes that the edges are better represented. However, some blocking artifacts are visible. The double smooth s-o wedgelets reduce slightly that problem. 


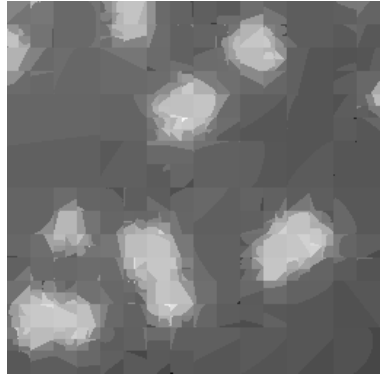

(a)

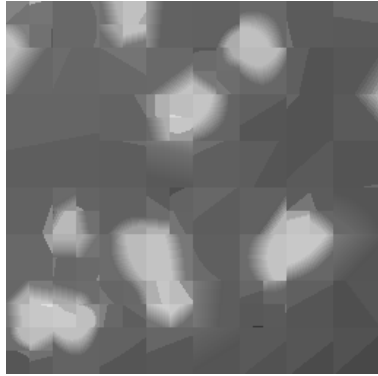

(b)

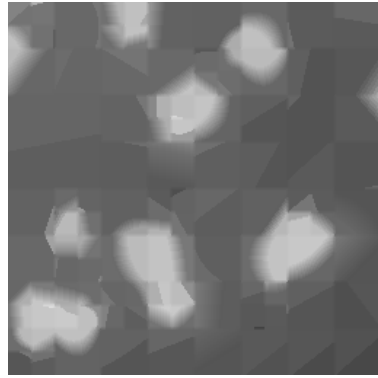

(c)

Fig. 4. Sample image (contaminated by Gaussian noise with variance equals to 0.015) denoised by: (a) s-o wedgelets, (b) smooth s-o wedgelets, (c) double smooth s-o wedgelets $\left(R_{S}=1\right)$

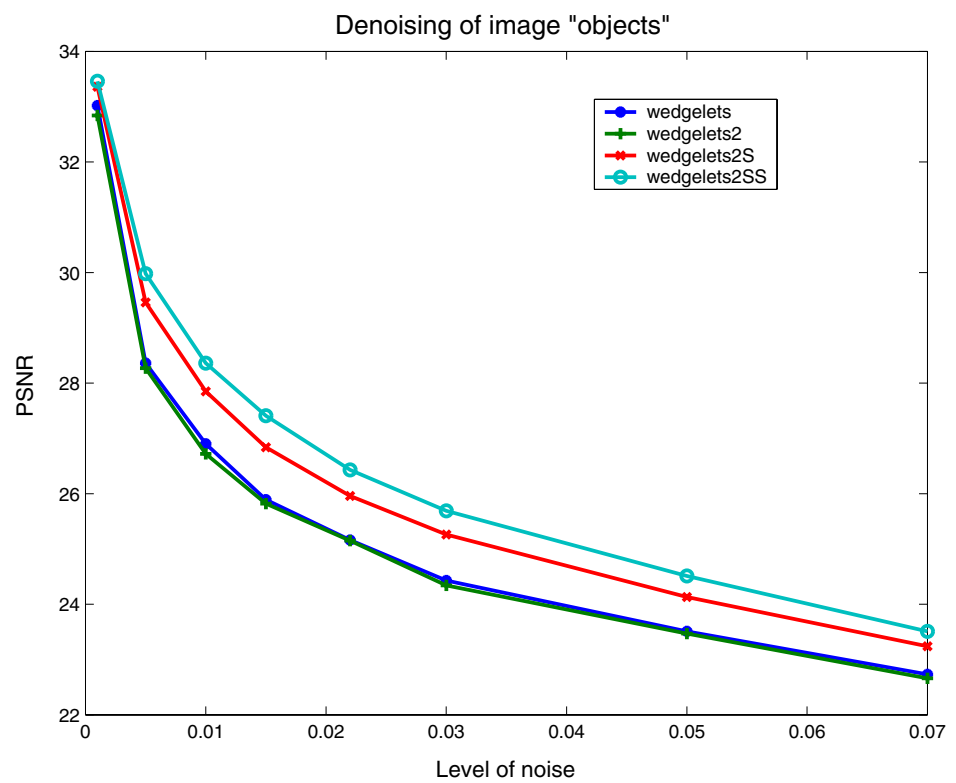

Fig. 5. Typical level-of-noise-PSNR dependency for the presented methods

Finally, in Fig. 5 there is presented typical dependency between four described methods of denoising. The plot was generated for image "objects", but for the remaining images the dependency is very similar.

\section{Summary}

In the paper smooth s-o wedgelets and their additional postprocessing have been introduced. Though the postprocessing step is well known and used in different 
approximation (estimation) methods based on quadtrees or similar image partitions it was never used in wedgelets-based image approximations (estimations), especially in denoising. In the case of images with smooth geometry it substantially improves the results of denoising, both — visually and computationally.

By comparing of denoising methods based on classical wavelets and wedgelets one can conclude that the former ones give better visual quality of reconstruction than the latter ones. But it is quite opposite if we consider computational quality. In fact, both of them have disadvantages - wavelets-based methods tend to smooth sharp edges and wedgelets-based methods produce false edges. The proposed method seems to overcome both inconveniences thanks to the adaptivity and postprocessing, respectively.

\section{References}

1. Donoho, D.L., Johnstone, I.M.: Ideal Spatial Adaptation via Wavelet Shrinkage. Biometrica 81, 425-455 (1994)

2. Donoho, D.L.: Denoising by Soft Thresholding. IEEE Transactions on Information Theory 41(3), 613-627 (1995)

3. Donoho, D.L., Vetterli, M., de Vore, R.A., Daubechies, I.: Data Compression and Harmonic Analysis. IEEE Transactions on Information Theory 44(6), 2435-2476 (1998)

4. Candès, E.: Ridgelets: Theory and Applications, PhD Thesis, Departament of Statistics, Stanford University, Stanford, USA (1998)

5. Candès, E., Donoho, D.: Curvelets - A Surprisingly Effective Nonadaptive Representation for Objects with Edges Curves and Surfaces Fitting. In: Cohen, A., Rabut, C., Schumaker, L.L. (eds.). Vanderbilt University Press, Saint-Malo (1999)

6. Mallat, S., Pennec, E.: Sparse Geometric Image Representation with Bandelets. IEEE Transactions on Image Processing 14(4), 423-438 (2005)

7. Donoho, D.L.: Wedgelets: Nearly-Minimax Estimation of Edges. Annals of Statistics 27, 859-897 (1999)

8. Donoho, D.L., Huo, X.: Beamlet Pyramids: A New Form of Multiresolution Analysis, Suited for Extracting Lines, Curves and Objects from Very Noisy Image Data. In: Proceedings of SPIE, vol. 4119 (2000)

9. Willet, R.M., Nowak, R.D.: Platelets: A Multiscale Approach for Recovering Edges and Surfaces in Photon Limited Medical Imaging, Technical Report TREE0105, Rice University (2001)

10. Starck, J.-L., Candès, E., Donoho, D.L.: The Curvelet Transform for Image Denoising. IEEE Transactions on Image Processing 11(6), 670-684 (2002)

11. Demaret, L., Friedrich, F., Führ, H., Szygowski, T.: Multiscale Wedgelet Denoising Algorithm. In: Proceedings of SPIE, Wavelets XI, San Diego, vol. 5914, pp. 1-12 (2005)

12. Lisowska, A.: Image Denoising with Second-Order Wedgelets. International Journal of Signal and Imaging Systems Engineering 1(2), 90-98 (2008)

13. Lisowska, A.: Effective Coding of Images with the Use of Geometrical Wavelets. In: Proceedings of Decision Support Systems Conference, Zakopane, Poland (2003) (in Polish)

14. Lisowska, A.: Geometrical Wavelets and their Generalizations in Digital Image Coding and Processing, PhD Thesis, University of Silesia, Poland (2005) 